\title{
PHOTOGRAPHS OF CORONAL STREAMERS
}

FROM A ROCKET ON 9 MAY 1967*

R. Tousey, G. D. Sandlin, and M. J. Koomen

E. O. Hulburt Center for Space Research

U. S. Naval Research Laboratory

Washington, D. C. 20390 USA

$$
R-09-029-036
$$

Presented at the

International Astronomical Union Meeting

Symposium No. 35: THE STRUCTURE AND DEVELOPMENT

OF SOLAR ACTIVE REGIONS

Budapest, Hungary

October 1967

* Supported by the National Aeronautics and Space Administration
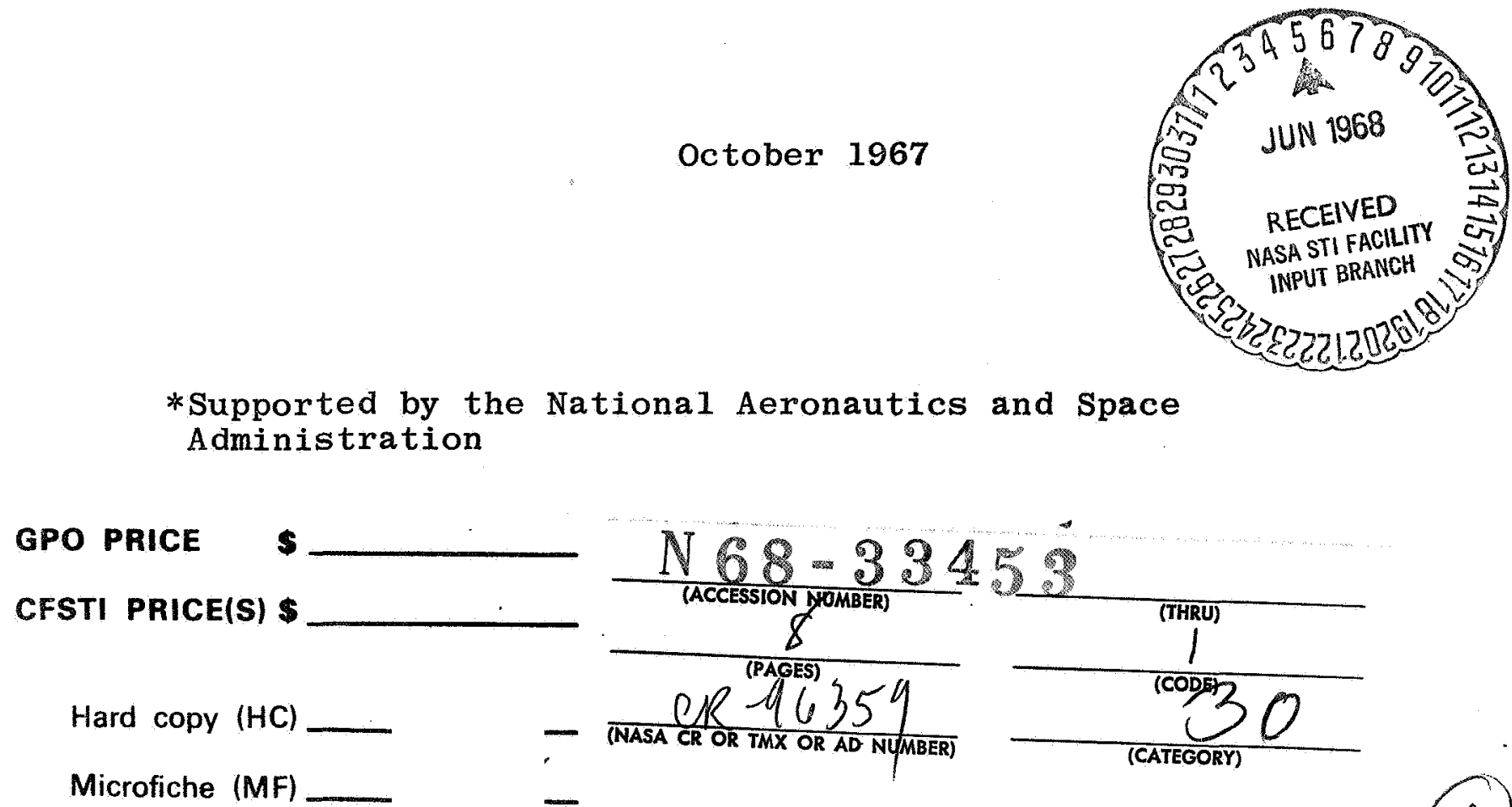


\title{
PHOTOGRAPHS OF CORONAL STREAMERS
}

F'ROM A ROCKET ON 9 MAX 1967*

\author{
R. Tousey, G. D. Sandlin, and M. J. Koomen \\ E. O. Hulburt Center for Space Research \\ U. S. Naval Research Laboratory \\ Washington, D. C. 20390
}

The most recent flight of the U. S. Naval Research Laboratory's white light coronagraphs took place on 9 May 1967. Two externally-occulted, small Lyot coronagraphs were built into the instrument compartment of the biaxial pointingcontrol section of an Aerobee-150 rocket. The instrument and some earlier results have been described by Tousey (1965), Purcell, Tousey, and Koomen (1967), and Koomen, Tousey, and Seal (1967). In the present noteattention is directed to the straight character of the streamers and to the possible origins of the three which appear to come from regions not far Irom the North Pole.

Simultaneous photographs from the two instruments are reproduced in Figure 1 , as unretouched, positive prints. The dark central region is the shadow cast by the external occulter; the dark sectors, in the INW of one and in the $\mathrm{SE}$ of

* Supported by the National Aeronautics and Space Administration 
the other, are the shadows of the arms supporting each occulter. At the very center of one a white disc has been introduced to show the location and size of the sun. The other instrument became slightly misaligned during launch, which accounts for the bright crescent at the edge of the occulter's shadow. The decrease in brightness of the corona and streamers with increasing $R$ was compensated by the vignetting action of the external occulter. The useful field covered the range 3 to $9 R_{\odot}$ in the plane perpendicular to the line of sight.

A few artifacts are present, caused by specks on a lens, and the short, bright streak in the NW of the one photograph is the trail of a sunlit particle just beyond the occulter. Mercury is seen almost out of the field in the west, and drawn into a short arc by the precession-caused rotation of the instrument around the solar vector.

The small, black disc east of the sun is the moon. The rocket was launched intentionally on 9 May at 1618 UT in order to have the moon in the field of view. This aspect of the program has been described by Koomen, Tousey, and Seal (1967). The black character of the shadow of the occulting disk and of the moon shows that the instrumental stray light level was negligible. The moon's features are clearly visible in earthshine on the original film. 
There are many conspicuous coronal streamers, especially at low solar latitudes. A11 of the streamers are straight; therefore, the range beyond $3 R_{\odot}$ is largely outside the region where the corona is controlled by complex magnetic fields. It will be difficult to locate the origins of the low-latitude streamers because of the great amount of activity and because of the overlapping and merging of the streamers. The streamers at high north latitudes can be studied in more detail. The NE streamer is single and appears to form a $21^{\circ}$ angle with the solar axis; the NW streamer is double with angles of $20^{\circ}$ and $27^{\circ}$ to the axis. It appears unlikely that they really originated at high latitudes because there were no large prominences in suitable positions and because sunspots and intense active centers were not found at those latitudes. They could very well have come from low latitude centers of activity on the front or back side and thus be seen in projection. On the front side, however, there were no features from which the streamers conceivably could have arisen.

An investigation was made to determine whether or not intense active regions on the back side might have served as origins for these streamers. Examination of the Ca-K spectroheliograms for 25 April and 23 May, fourteen days 
earlier and later than 9 May, showed plages in favorable locations, at latitudes where the solar rotation period is about 28 days. These regions were very large and intense, containing large spots at coordinates $31^{\circ} \mathrm{E}, 26^{\circ} \mathrm{N}$ under plage \#8785, at $19^{\circ} \mathrm{E}, 35^{\circ} \mathrm{N}$ under plage \#8793 which first appeared on $28 \mathrm{April}$, and at $22^{\circ} \mathrm{W}, 23^{\circ} \mathrm{N}$ under plage \#8778. On 24 May the same regions persisted on the sun, remaining very intense and at roughly the same positions but having grown markedly in area; the eastern region, designated \#8818 in this rotation, covered about 8500 millionths of the hemisphere, and the western region covered about 2500 millionths of the hemisphere. The eastern region at $247^{\circ}$ longitude was the most active region on the sun in May, and was the site of the proton flare events in the last week of May. Average positions for these regions were taken and transferred to the back of the sun. Their locations and approximate forms are indicated on the white disc in Figure 1 as a view seen through the sun.

A calculation was made of the apparent angle between each streamer and the solar axis, using the assumed positions of the active centers, making the assumption that the streamers were radial, and taking into account the tilt angle 
of the sun and the perspective. The following table gives the results.

TABLE I

APPARENT ANGLE BETWEEN STREAMER AND SOLAR NORTH

\begin{tabular}{lccc}
\hline Plage \#/Streamer & $8778 / \mathrm{NE}$ & $8785 / \mathrm{NW}$ & $8793 / \mathrm{NW}$ \\
\hline Observed & $21^{\circ}$ & $27^{\circ}$ & $20^{\circ}$ \\
Calculated & $20^{\circ}$ & $29^{\circ}$ & $17^{\circ}$ \\
\hline
\end{tabular}

These values are in very good agreement, in consideration of the errors of measurement and of the uncertainties involved in estimating the centers of the plages.

The conclusion is that these streamers came from active centers that were $50^{\circ}$ to $53^{\circ}$ heliocentric angle behind the limb, and that they were radial. Because of perspective, they were realiy recorded to about $15 R_{\odot}$. Although they were favorably located to show any twisting, there is no indication of a "garden hose" effect because they are straight, and project inward along radii passing through the large centers of activity present on the rear side.

We are greatly indebted to Dr. Helen Dodson-Prince, and Miss E. Ruth Hedeman of the McMath-Hulbert Observatory for making available their solar data and for their discussions of the histories of the active regions. 
REFERENCES

Koomen, M. J., Tousey, R., and Seal, R. T. Jr.: 1967, to be published in SPACE RESEARCH VIII.

Purce11, J. D., Tousey, R., and Koomen, M. J.: 1967, to be published in SPACE RESEARCH VIII.

Tousey, R.: 1965, Ann. d'Astrophys. 28, 600 . 


\section{FIGURE CAPTION}

Simultaneous photographs of the white light corona, made with a pair of externally occulted Lyot coronagraphs, flown from the White Sands Missile Range on 9 May 1967 at 1618 UT. Introduced into the central shadow cast by the occulter of one instrument is a disk showing the size and location of the sun, and the approximate locations and shapes of the plages, believed to be on the back side of the sun. The sharp black disc is the moon, and the bright, short arc at the edge of the field is Mercury. The bright arc in the left image is evidence of slight misalignment; the streak in the right image is a particle trail, out of focus because it was not far beyond the occulter. 


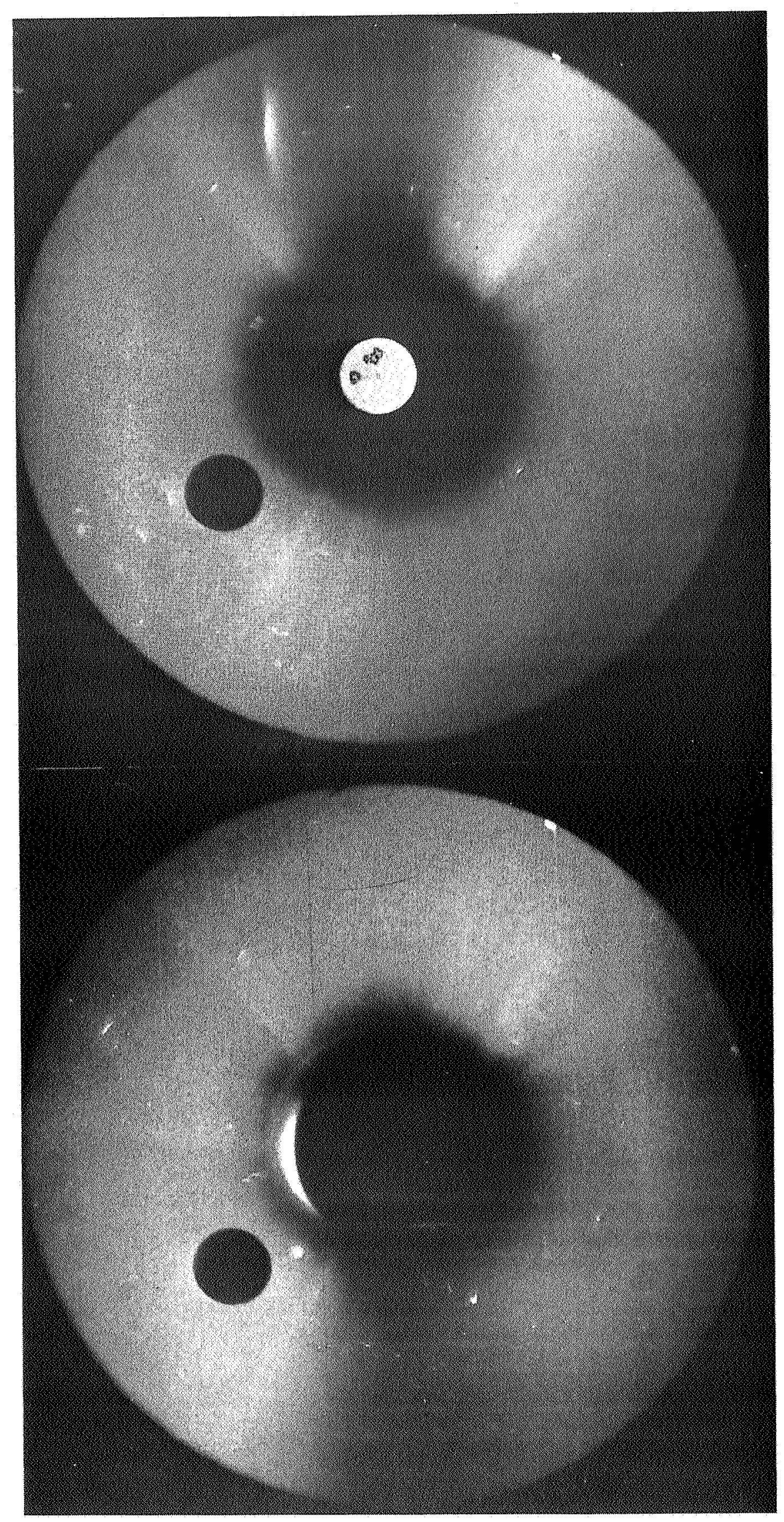

龒 